\title{
EFICIENCIA Y EQUILIBRIO EN LOS MERCADOS DE CAPITALES
}

\author{
SONSOLES HUERTA DE SOTO*
}

En este breve artículo pretendo dar respuesta a la pregunta de si los mercados de capitales son o no eficientes. Para ello comienzo criticando uno de los pilares fundamentales de los economistas clásicos, el equilibrio paretiano, y planteo como alternativa la eficiencia dinámica. Por último, procedo a la aplicación concreta de lo expuesto en el mercado de capitales.

La gran pregunta entre los teóricos de las inversiones financieras y los prácticos de las mismas ha sido y sigue siendo si los mercados de capitales son eficientes.

Es preciso, pues, partir del concepto de eficiencia.

\section{CONCEPTO DE EFICIENCIA ESTÁTICA}

La gran mayoría de los economistas actuales entiende la eficiencia como la minimización del despilfarro de recursos económicos o, lo que es lo mismo, la maximización de la satisfacción de las necesidades sometida a la restricción de recursos y tecnología dados. Éste es el concepto estático de eficiencia económica que con carácter predominante se ha utilizado en la ciencia económica y que alcanza su culmen con la teoría del equilibrio de Pareto. Sin embargo, estos criterios tradicionales de

(*) Universidad Complutense (Madrid). 
eficiencia asignativa de origen paretiano son insuficientes para ser aplicados como criterio normativo a la rica dinámica de las instituciones sociales que se dan en la vida real.

Según el enfoque paretiano, un sistema económico se encuentra en una situación de eficiencia (estática) si no es posible mejorar a alguien sin empeorar a otro. Los teóricos paretianos creen posible alcanzar esta situación de equilibrio, y, en términos muy generales, consideran que el equilibrio del mercado es el resultado de la intersección entre la oferta y la demanda agregadas (de todos los mercados existentes en una economía). La intersección entre oferta y demanda determina el llamado precio de equilibrio o valor intrínseco de un bien. Elabora toda una compleja teoría basada en axiomas de carácter matemático en un intento de inducir las leyes que rigen la ciencia económica. Toda su teoría parte de lo que él denomina curvas de indiferencia o utilidad, a partir de las cuales elabora las curvas de oferta y demanda del mercado. Es precisamente en la construcción de estas curvas donde radica el error de Pareto, cayendo toda su teoría sobre el equilibrio por su propio peso. Suponen una caricaturización de la realidad, al simplificarla hasta tal punto que dejan de reflejarla.

En primer lugar, son un instrumento manifiestamente insuficiente para reflejar la rica y compleja realidad. En la vida real un individuo no elige entre dos bienes, sino entre los cientos y miles que hay en el mercado. El primer paso para la construcción de las curvas de indiferencia es preguntar al sujeto qué combinaciones cuantitativamente variables de dos bienes le son «indiferentes» y por ello igualmente importantes. Las respuestas, según Pareto, constituirían una serie muy larga ad infinitum de combinaciones. Pero, y esto es lo relevante, ello sería sólo una parte pequeñísima del experimento. Tendría que repetirse para tres bienes, cuatro bienes, etc., cubriendo todas las posibles combinaciones entre los mismos y dando lugar a un número infinito de combinaciones para todos 
los bienes del mercado. Se tendrían series infinitas de combinaciones indiferentes de pan y vino, de pan y carne, de vino y carne, etc., formando curvas de indiferencia de forma tridimensional para tres bienes y de forma inimaginable para $n$ bienes.

A través de las curvas de indiferencia es imposible, por tanto, plasmar las posibles combinaciones indiferentes de bienes que se dan en la economía real. Es un instrumento tan simplista de la realidad, omite tanta información existente, que la deforma.

En segundo lugar, se parte de que las curvas de indiferencia son continuas; otro error grave, ya que los bienes no son infinitesimalmente divisibles.

Pero la principal debilidad de las curvas de indiferencia es que suponen la ilimitada capacidad de sustitución de los diferentes bienes para la satisfacción de múltiples tipos de necesidades (hambre, sed). Sin embargo, una vez más, el análisis de la realidad demuestra que los bienes sólo pueden ser sustituidos dentro de límites muy estrechos, dada la multiplicidad jerárquica de necesidades particulares, numerosas y cualitativamente distintas, donde la satisfacción de una no puede ser reemplazada por la satisfacción de otra. Entre los humanos civilizados, el objetivo final de la economía no es la satisfacción de especies de necesidades (en esta idea se basa la construcción de las curvas de indiferencia), sino de gustos y placeres altamente diferenciados. En palabras de Rothbard ${ }^{1}$, la falacia crucial es que la indiferencia no puede ser base para la acción. Si el hombre fuese realmente indiferente entre dos alternativas, no podría elegir entre ellas y la elección no se revelaría en acción, el hombre no actuaría. A la economía como ciencia praxeológica, basada en la acción humana, no le interesa valorar

1 Murray N. Rothbard, Man, Economy, and State, Ludwig von Mises Institute, Auburn, Alabama, 2001, pp. 265-268. 
ni analizar las preferencias psicológicas de los individuos (la escala de valores interna de cada uno), sino la escala de valores reveladas por la acción. Mediante la acción siempre se revela una preferencia (jamás una «indiferencia»).

Las curvas de indiferencia son incapaces de describir el mundo real. Son relaciones básicas imaginarias entre bienes, vinculadas a través de relaciones totales irreales (indiferencia) con una situación hipotética e inexistente de equilibrio. Luego los precios y correlaciones de precios derivadas de esta construcción no son los del mundo real. Por tanto, debemos concluir que las curvas de oferta y demanda que podemos obtener a partir de las curvas de indiferencia no son reales, son una ficción, y el punto de equilibrio definido por éstas no es veraz, no existe.

El problema es que es precisamente el concepto de eficiencia estática y de equilibrio paretiano el que se ha impuesto en la economía y el que se enseña en las universidades y se aplica en el análisis de los mercados, por ejemplo, de los mercados financieros. La concepción estática de la economía reduce el concepto de eficiencia económica a un mero problema técnico de maximización, y la economía no es una ciencia matemática exacta, la economía es esencialmente dinámica e «imperfecta» (en el sentido de no encontrarse jamás «en equilibrio»; así, no existe ni existirá nunca un mercado «perfecto»).

\section{TEORÍA DE LA EFICIENCIA DINÁMICA}

El concepto de eficiencia dinámica está íntimamente unido al concepto de función empresarial. Ésta se puede definir como la capacidad típicamente humana para darse cuenta de las oportunidades de ganancia que surgen en el entorno, actuando en consecuencia para aprovecharse de las mismas. Las características más importantes de la función empresarial son: 
1. La función empresarial siempre genera nueva información. Todo acto empresarial supone el descubrimiento de una información nueva que antes no tenía el actor. Esta información la crean los propios empresarios en el momento en que la conciben en su mente y la concepción de cada empresario será distinta. La información es así subjetiva, única, dispersa en cada mente humana, y muy difícilmente articulable de manera formalizada. Es una oportunidad de ganancia que antes había pasado inadvertida, concebida en una mente humana en un momento determinado único.

2. La función empresarial es esencialmente creativa. Todo desajuste social se plasma en una oportunidad de ganancia que queda latente de ser descubierta (creada) por los empresarios. El empresario que la descubre crea una información en su mente que antes no existía, cuya materialización en un acto empresarial da lugar a la obtención de un beneficio empresarial puro.

3. La función empresarial transmite información (expresa cuál es el desajuste y su solución).

4. La función empresarial es coordinadora (elimina desajustes).

5. La función empresarial es competitiva. El proceso empresarial es, ante todo, un proceso de rivalidad netamente competitivo, ya que, una vez creada y aprovechada una oportunidad de ganancia en un tiempo y lugar concreto y por un empresario determinado, ya no puede ser creada y aprovechada esa misma oportunidad por otro.

6. El proceso empresarial nunca se detiene ni agota, ya que el acto empresarial crea nueva información que da lugar siempre a la creación de nuevos desajustes y oportunidades de ganancia. 
Por tanto, en un sentido dinámico, un sistema económico sería tanto más eficiente cuanto más y mejor impulse la creatividad y la coordinación empresarial. Lo importante para lograr la eficiencia dinámica es descubrir y crear continuamente nuevos fines y medios, impulsando la coordinación; siendo consustancial a este concepto de eficiencia la creación constante de nuevos desajustes económicos y sociales. Un cierto despilfarro es, por tanto, inevitable y necesario en toda economía de mercado. $\mathrm{O}$, expresado gráficamente: partiendo del concepto estático, una economía es eficiente cuando se encuentra en la frontera de posibilidades de producción; desde el punto de vista dinámico, una economía es eficiente cuando la frontera de posibilidades de producción avanza en continua expansión hacia la derecha. Luego puede considerarse que la dimensión dinámica engloba a la estática.

Es muy ilustrativa la concepción de Schumpeter del proceso empresarial como una fuerza explosiva que distorsiona el orden preexistente. De lo que se olvidó Schumpeter, en palabras de Jesús Huerta de Soto $^{2}$, es de que esta misma fuerza que impulsa la destrucción creadora tiende a coordinar el sistema económico, haciendo que el «big bang» social sea tan armonioso como sea posible en cada circunstancia histórica.

Llegados a este punto, estamos en condiciones de aplicar la teoría de la eficiencia dinámica a los mercados de capitales.

2 Huerta de Soto, Jesús, "La teoría de la eficiencia dinámica», en Procesos de Mercado: Revista Europea de Economía Política, vol. 1, n. ${ }^{\circ}$ 1, primavera 2004, pp. 35-36. 


\section{EFICIENCIA DINÁMICA EN LOS MERCADOS DE CAPITALES}

Las tres hipótesis de eficiencia que se utilizan en el análisis financiero de los mercados se construyen en base al concepto de eficiencia estática, que como ya hemos visto tiene un rango de importancia muy inferior al de eficiencia dinámica. La hipótesis débil supone que cada título refleja totalmente la información contenida en la serie histórica de precios, es decir toda la información pasada. Un inversor podrá «batir al mercado» utilizando la información hecha pública por las empresas y la información privilegiada, ya que la información pasada se supone conocida por todos. La hipótesis intermedia sostiene que los precios no sólo reflejan toda la información pasada sino también toda la información hecha pública por las empresas. La única forma de lograr un rendimiento superior es a través de la información privilegiada. Por último, según la hipótesis fuerte los precios reflejan absolutamente toda la información, ya sea pasada, pública o privada. Ningún inversor puede «batir al mercado», es decir, el mercado es «perfecto». Hasta ahora el estudio de la eficiencia de los mercados financieros se ha enfocado erróneamente.

El concepto de eficiencia y equilibrio que se maneja por los teóricos financieros es el estático. Las tres hipótesis analizan tres niveles evolutivos del mercado perfectamente competitivo. La débil describe un mercado claramente ineficiente y la fuerte un mercado perfectamente competitivo. Pero desde el punto de vista dinámico un mercado perfectamente competitivo no es sólo irreal e inalcanzable, sino que sería un "no mercado». Si no existen posibilidades de ganancia no existe el mercado. Carece de sentido analizar, ni tan siquiera cuestionarse, si los mercados de capitales pueden funcionar en competencia perfecta, alcanzar el equilibrio, como si esto fuese lo deseable; porque un mercado en equilibrio sería, por definición, un no mercado 
(puesto que éste no es sino un proceso empresarial de continua creación de nuevas oportunidades de ganancia). Un mercado «perfecto» es una contradicción en los términos, como las de círculo cuadrado, nieve caliente o prostituta virgen.

Otro error importante es la consideración de la información económica como información objetiva. La información económica, como ya se ha dicho, no es sino subjetiva. Los títulos valores que se intercambian no reflejan ninguna información objetiva (salvo su propio precio «histórico») ni tienen valor intrínseco o precio de equilibrio. La información relevante se crea cada vez que un especulador concibe una nueva posibilidad de ganancia en el mercado de capitales. Los precios históricos de los valores, los datos de las empresas y la llamada «información privilegiada» no es información económica hasta que no se interioriza por un individuo concreto y da lugar a la creación de una oportunidad de ganancia en una mente determinada. En la economía no existen datos objetivos independientes (aislados) de su interpretación subjetiva por una mente humana, y es esta interpretación personalísima la única relevante económicamente; a partir de la ella nace una idea que se plasma en un acto empresarial. La información así concebida es en puridad, o sea totalmente, asimétrica; nadie tiene exactamente la misma información subjetiva (cada mente crea una información distinta, percibe la realidad de forma diferente).

Por tanto, ni la información «objetiva» se refleja unívocamente en los títulos ni la oportunidad de ganancia se encuentra en el tiempo de ajuste entre el precio de mercado y el supuesto valor intrínseco del título. No depende del ajuste precio-valor, sino de la función empresarial, la creatividad del empresario, la capacidad de concebir nuevas ideas en cada situación de tiempo y lugar, y la capacidad coordinadora del acto empresarial.

Desde este punto de vista, carece de sentido el análisis técnico o fundamental para predecir los precios futuros de los 
valores. Los precios de los títulos son impredecibles (como las ideas del ser humano), surgen de la oferta y demanda individual de títulos que hace cada inversor basándose en las oportunidades de ganancia descubiertas por él.

Podemos concluir, por tanto, que el mercado de capitales tiende a ser dinámicamente eficiente, entendido como un proceso de constante creación y descubrimiento de oportunidades de ganancia y de desajustes que, regido por el papel coordinador del acto empresarial, hace avanzar a la economía, con un ritmo y hacia un futuro desconocidos.

\section{BIBLIOGRAFÍA}

Mises, Ludwig Von (2004), La Acción Humana: Tratado de Economía, 7. ${ }^{\text {a }}$ edición, Unión Editorial, Madrid.

MAYER, Hans (otoño 2004), «El valor cognitivo de las teorías funcionales del precio», en Procesos de Mercado: Revista Europea de Economía Política, Unión Editorial, pp. 141-261.

HuerTa DE Soto, Jesús (primavera 2004), «La teoría de la eficiencia dinámica», en Procesos de Mercado: Revista Europea de Economía Política, Unión Editorial, pp. 11-71.

Rothbard, Murray N. (2001), Man, Economy, and State, Ludwig Von Mises Institute. Scholar's Edition [2004]. 